
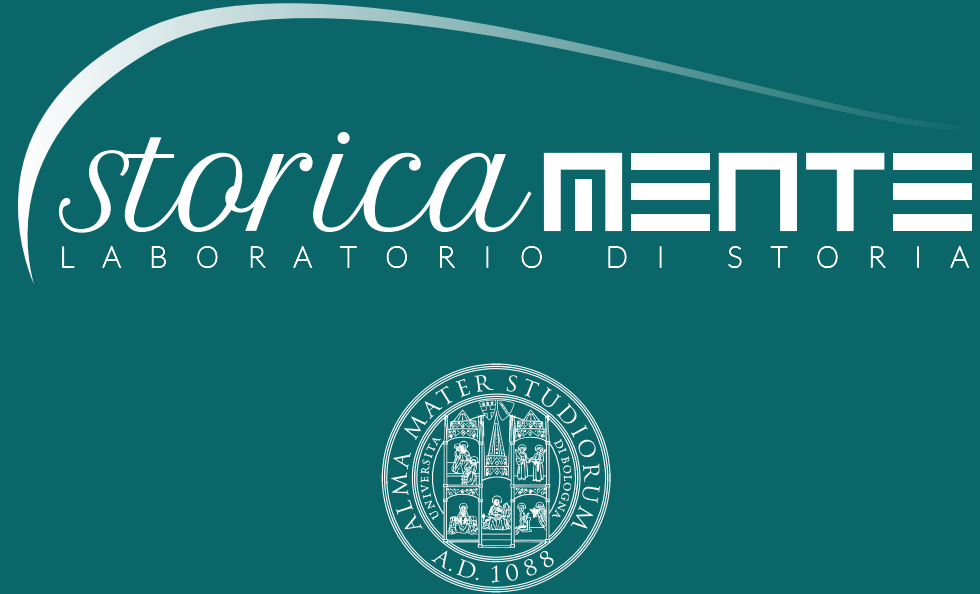

ALMA MATER STUDIORUM

Università di Bologna

Dipartimento di Storia Culture Civiltà

COMUNICARE STORIA

LA STORIA A SCUOLA OGGI.

INSEGNARE STORIA

NELLA SCUOLA PRIMARIA 


\section{STORICAMENTE.ORG}

\section{Laboratorio di Storia}

Cecilia Ricci, Stefano Colavecchia

Storia e didattica della storia nella formazione iniziale dei docenti

Numero $15-2019$

ISSN: $1825-411 X$

Art. 53

pp. $1-16$

DOI: $10.12977 /$ stor799

Editore: BraDypUS

Data di pubblicazione: $18 / 10 / 2020$

Sezione: Comunicare storia: "La Storia a scuola oggi. Insegnare storia nella scuola primaria" 


\title{
Storia e didattica della storia nella formazione iniziale dei docenti
}

\author{
CECILIA RICCI, STEFANO COLAVECCHIA
}

Univ. Molise, Dipartimento di Scienze Umanistiche, Sociali e della Formazione

The paper presents the experiences of the two Authors on the teachings of Ancient History with elements of History Didactics (Cecilia Ricci) and Modern History (Stefano Colavecchia). The account of the experiences is preceded by a brief introduction on some delicate and largely unresolved issues.

Un problema strutturale: la didattica della storia nei corsi delle università italiane

In questo articolo ${ }^{1}$ presenteremo le nostre esperienze, che riguardano rispettivamente gli insegnamenti di storia antica con elementi di didattica (Cecilia Ricci) e di storia moderna (Stefano Colavecchia), presso il corso di studi in Scienze della formazione primaria (d'ora in avanti Sfp) dell’Università degli Studi del Molise, facendole precedere da una breve

A Cecilia Ricci si devono le prime tre sezioni: "Un problema strutturale", "Gli "snodi periodizzanti»", "L'esperienza di Unimol per la storia antica”. A Stefano Colavecchia si deve l'ultima sezione: "L'esperienza di Unimol per la storia moderna: storia e digital humanities, una proposta didattica". 
premessa su alcune questioni sensibili, molto discusse e, in larga parte, ancora irrisolte (Cecilia Ricci).

Definiremmo la prima questione un problema "strutturale" che impone una riflessione da parte di tutti coloro che insegnano discipline storiche e, possibilmente in tempi ragionevoli, l'individuazione del modo più opportuno di procedere alla sua soluzione.

Il percorso formativo del corso di studi in Sfp è articolato, come si sa, in attività distinte tra insegnamenti con esami, laboratori e tirocini. Per farmi un'idea di quali insegnamenti di Storia sono previsti, ho selezionato, a scopo puramente esemplificativo, cinque università italiane del nord, del centro e del sud della penisola (Università degli Studi di Torino, Università degli Studi di Verona, Unimore - Università degli Studi di Modena e Reggio Emilia, Università degli Studi di Roma Tre, Università degli Studi di Bari), consultando nei rispettivi siti il link "Piano degli Studi" in Sfp.

Scorrendo l'elenco degli insegnamenti di storia e di didattica attivati, ci si accorge subito che domina un'assoluta difformità, con un grande assente, l'insegnamento di didattica della storia. Tale difformità solo in parte trova una valida spiegazione nell'autonomia universitaria ed è più spesso dovuta all'organico a disposizione dei singoli atenei e al (sovrac) carico orario dei docenti delle discipline storiche, che si trovano im-

URL dei piani di studio in Sfp di cinque università italiane. Per tutte, il piano di studio si riferisce all'a.a. 2019-2020, tranne che per l'ateneo di Bari (online è reperibile il piano 2018-2019). Per tutti i siti, l'ultima consultazione risale 17.03.2020:

Torino:

https://formazioneprimaria.campusnet.unito.it/do/corsi.pl/ Search?title=Corsi $\% 20 \mathrm{e} \% 20$ moduli;first $=300$;

Verona: http://www.dsu.univr.it/?ent=pd\&cs=792;

Unimore: https://offertaformativa.unimore.it/corso/insegnamenti?cds_cod=83252\&pds_cod $=0 \&$ coorte $=2019 \&$ durata $=5 \&$ anno_corrente $=2019 \&$ aa_off_id $=0$;

Roma Tre: http://www.uniroma3.it/didattica-programmata/0d2a38bc-a92b-49e8bba1-0635abd1a5dc--1954360a-91a7-406b-a654-b3fd71c48c93/];

Bari [https://www.uniba.it/corsi/scienze-formazione-primaria/studiare/offerta-formativa/offerta-formativa-2018-19.pdf/view]. 
possibilitati ad attivare altri corsi e/o non possono mutuarli per evidenti ragioni di cfu, e prima ancora di metodi e finalità didattici.

Guardando a questa situazione con ottimismo, si direbbe che non mancano la consapevolezza di quel che sarebbe opportuno fare né la volontà di procedere nella giusta direzione: è la contingenza a imporre decisioni, scelte o rinunce che tuttavia inevitabilmente si ripercuotono sugli studenti (e futuri maestri) che "subiscono" un trattamento differenziato. Se invece lasciamo da parte l'ottimismo, l'assoluta deregulation nelle scelte didattiche, nascosta dietro l'alibi dell'autonomia universitaria, svela un gravissimo deficit di progettazione prima e monitoraggio a livello centrale poi.

Ma entriamo nello specifico. La storia che va insegnata nella primaria va dall'ominazione al tardoantico. Se però diamo una scorsa attenta agli insegnamenti di Storia presenti nei piani di studio dei corsi di Sfp delle università italiane (non solo naturalmente quelle precedentemente indicate), fatto salvo il caso di pochissimi grandi atenei dove l'alto numero dei docenti lo consente, il quadro generale è drammaticamente chiaro:

1. la preistoria è quasi del tutto assente;

2. la storia antica è poco presente; chi la insegna è spesso un solo docente per la storia greca e quella romana e mai, a quel che mi risulta, un docente di storia del vicino Oriente antico;

3. pochissime università hanno organizzato corsi integrati che dall'antichità arrivano al contemporaneo;

4. spesso si trovano solo corsi (talvolta anche solo uno) di storia moderna o contemporanea;

5. in molti casi, gli insegnamenti di storia sono a scelta dello studente (storia greca o storia romana, storia medievale o storia antica, storia moderna o storia contemporanea e così via).

La situazione, oltre ai motivi sopra ricordati (deregulation, mancanza di coordinamento, scarsità di docenti a disposizione...) è in parte anche conseguenza del numero inadeguato di crediti a disposizione per le storie e della debolezza generale di tutta l'area storico-geografica, come 
e più di altri insegnamenti disciplinari. Mancando un coordinamento, il rischio è di lasciare molto (troppo) i docenti da soli, costretti a operare scelte individuali, anche se forte è il bisogno di avviare un confronto e uno scambio di idee, dubbi, esperienze.

Un primo passo per ovviare a questo stato (critico) di cose potrebbe essere la creazione di un coordinamento tra chi insegna storia o didattica della storia nei corsi di Sfp degli atenei italiani, stabilendo un contatto, per consolidare la rete, con la Commissione didattica del coordinamento delle società storiche, attualmente presieduta da Walter Panciera dell'Università di Padova, e con la neonata Commissione per la didattica della storia nelle scuole presieduta da Andrea Giardina, già docente alla Scuola Normale di Pisa ${ }^{3}$.

\section{Gli «snodi periodizzanti» e la programmazione didattica}

Una seconda questione riguarda i contenuti degli insegnamenti storici e la programmazione didattica. Sappiamo bene che le indicazioni nazionali, anche per la scuola primaria, sottolineano come urgenza l'aggiornamento dell'insegnamento della storia sui risultati della ricerca storica più recente.

La questione delle questioni è come conciliare la necessità di soffermarsi sugli «snodi periodizzanti della vicenda umana» con la nuova scansione cronologico-sequenziale che, dal terzo al quinto anno, prevede di organizzare un percorso che dalla comparsa dell'uomo arriva al $\mathrm{V}$ secolo d.C. ${ }^{4}$

"Una commissione per la didattica della storia nelle scuole con presidente Andrea Giardina.” Normalenews, 29 gennaio 2020 (https://normalenews.sns.it/una-commissione-per-la-didattica-della-storia-nelle-scuole-con-presidente-andrea-giardina).

4 D.M. 16 novembre 2012, 254: "Identità, memoria e cultura storica". 
Di fatto, è il docente di un qualsiasi insegnamento di storia - presente nel piano di studi di un corso in SFP - a diventare lo snodo, di carne e sangue, dovendo fornire ai futuri maestri un'ipotesi di interpretazione dei dettami della riforma; o quella che i tedeschi chiamano una Praxisorientierung, una proposta di orientamento alla professione di docenti di storia (Hasberg 2019).

Credo che il senso di responsabilità e la paura di essere sopraffatti dalla quantità di informazioni da trasmettere abbia provocato, almeno in una prima fase, in tutti i docenti che si trovano in tale condizione, un senso di smarrimento.

La domanda che (certamente non solo io!) mi sono posta è: quali sono gli snodi periodizzanti o, se si preferisce, i passaggi chiave nello sterminato universo spazio-temporale della storia antica? $\mathrm{E}$ facile accorgersi che una risposta univoca non c'è e, al momento, quella che si può trovare è parziale, operativa, temporanea e occasionale. Personalmente ho avuto la sensazione di essere davanti a un bivio, con da una parte le occasioni e i pretesti per una riflessione sulla storia d'Italia attraverso le tracce materiali del passato, dall'altra pensando a come procedere alla selezione, sentendo forte il rischio di trascurare, nel mio percorso, alcuni dei passaggi essenziali, e sforzandomi di immaginare come sia possibile calare tutto ciò nella didattica modulare e in quella laboratoriale. Credo che, dopo anni d'incubazione, sia ormai arrivato il momento di avviare una riflessione sistematica su questa ipotesi di cronologia di base come singoli e, al più presto, in gruppi di lavoro a questo dedicati. Una volta inventati gli snodi, superando i sensi di colpa per consapevoli quanto inevitabili omissioni, occorre procedere dalla teoria alla pratica. La progettazione di curricoli di storia originali e sostanziosi è un'operazione complessa, che richiede l'individuazione e il ricorso a strumenti didattici appropriati. Anche qui lo sconforto prevale: non spetta a me parlarne, ma la manualistica a disposizione è tradizionale nell'organizzazione dei contenuti. E gli strumenti non sono solo i manuali, ma anche $\mathrm{i}$ testi che, prima ancora che gli alunni, guidino nel modo più 
agevole possibile i futuri maestri alla scoperta del patrimonio culturale, oltre ai servizi didattici di vario tipo, in primis quelli museali, presenti sul territorio (Donato, 2014).

\section{L'esperienza di Unimol per la storia antica}

Il corso di studi in Scienze della formazione primaria (Sfp) dell'Università degli Studi del Molise è giovane (2001-2002), e la Laurea magistrale a ciclo unico parte, come sappiamo, a seguito del decreto ministeriale 249 del 10 settembre 2010 .

Gli insegnamenti di storia hanno subito negli ultimi dieci anni continue rimodulazioni, con variazioni di denominazioni, numero di crediti, docenti dedicati e altro. Il clima che a lungo ha dominato - crediamo di non dire nulla di nuovo - è quello di una "dialettica vivace" tra la componente dei docenti di pedagogia e materie applicate da una parte, e di quelli delle materie cosiddette disciplinari dall'altra.

Per la storia antica, dopo una prima fase sperimentale, dal 2018-2019 viene proposto un modulo obbligatorio da $8 \mathrm{cfu}$, ripartiti in $6 \mathrm{cfu}$ di civiltà del mondo antico e 2 cfu di didattica. Le altre storie (medievale, moderna e contemporanea) sono a scelta ed esenti dai cfu di didattica. In tutti e quattro i casi, con o senza moduli di didattica, i crediti previsti dalla normativa sono evidentemente inadeguati rispetto alla mole di conoscenze e competenze previste negli obiettivi formativi. Ma naturalmente questo è un problema per tutti i percorsi di Sfp.

Il corso di storia antica funziona esclusivamente con attività didattica in presenza, finora senza ausilio di piattaforma, e-learning, ecc. Utilizzo le 48 ore di cui dispongo in questo modo: dopo un'introduzione sui problemi della didattica nella scuola, propongo un quadro storico di riferimento con la "mia" cronologia essenziale; nella parte finale del corso, la presentazione di un tema specifico apre uno spazio paralaboratoriale per domande storiche e per un ruolo più partecipativo degli studenti. 
Un'esperienza laboratoriale vera e propria, per ora, non c'è, ed è riservata alle laureande e ai laureandi che l'attivano con l'aiuto congiunto del docente e del tutor per il tirocinio. I temi scelti per i lavoro di tesi sono quelli che, per motivi di tempo, non si riesce a trattare nel corso: religione, vita sociale, storia locale, sport, spettacoli e, in qualche caso, ricerche sui temi didattici.

Nella prima parte del corso, che regolarmente travalica le 12 ore a disposizione, lo spazio della lezione frontale è ridotto al minimo. Si discute insieme o in gruppi di lavoro sulla priorità di conoscenze, capacità e abilità da ricercare. Dal confronto tra gli studenti, anche se in misura e modi diversi, regolarmente emergono le medesime priorità tra gli obiettivi da conseguire nel lavoro in classe con gli alunni: la conoscenza delle idee politiche, con l'acquisizione di un vocabolario per capire i fenomeni storici e i processi politici; la capacità di farsi opinioni, rafforzarle e dimostrarle, e di accrescere la propria autostima, in famiglia e nella società; la capacità di far parte con consapevolezza di strutture sociali, anche basilari, conoscendone le regole.

Per la parte disciplinare, da anni propongo un itinerario di storia istituzionale (sia per la parte greca che per quella romana) focalizzato sui mutamenti e i principali assetti politici: il mondo vissuto e raccontato da Omero, l'esperimento democratico ad Atene, l'oligarchia spartana, e, per Roma, l'esperienza monarchica, la costruzione del modello repubblicano, la "rivoluzione" augustea e i nuovi assetti tra Diocleziano e Costantino. Ho scelto dunque di concentrarmi sui grandi passaggi istituzionali: tirannidi, democrazie, oligarchie, monarchie, principati, imperi, dominati, ecc.

L'obiettivo dell'itinerario è l'acquisizione delle conoscenze di base sulle quali costruire un metodo di studio ai fini dell'esame, che verte su un programma ampio, e di lavoro nel futuro professionale.

Ogni snodo istituzionale viene argomentato cercando di stimolare reazioni, anche critiche, attraverso strategie semplici, quali ad esempio la proposta di analogie storiche, che sovente prendono spunto dalla situa- 
zione economica o militare odierna, dalle tematiche di genere o dalle migrazioni e le traspongono a ritroso nelle società greche e nelle città dell'impero romano, per verificare se e in che modo tali problematiche esistessero, e come e in che tempi venissero affrontate.

L'idea è quella di partire dai problemi o dalle grandi questioni dell'oggi e, parallelamente, dalle costanti storiche (morte, matrimoni, alimentazione, ecc.) per cercare di comprendere, per linee generali se non nei dettagli, la distanza o la vicinanza dei modi e degli strumenti di volta in volta adottati per affrontare i problemi.

Il salto indietro e lo scarto laterale, mantenendosi per quanto possibile accanto agli assi di storia verticale e di storia orizzontale, suscitano curiosità, non di rado difficoltà, ma sempre fanno riflettere sulle persistenze, sulle discontinuità, sulle rotture, e generano la consapevolezza che questo modo di procedere possa aiutare, in classe, i bambini a costruire e poi maturare competenze e abilità, a divertirsi nel fare e nel suggerire connessioni, e ancora, non da ultimo, a coltivare la suprema arte del dubbio.

Al termine di questa carrellata, come anticipato, ogni anno preparo un mini-modulo con un caso di studio, per estendere lo sguardo alla ricerca storica. Quest'anno il tema è la figura femminile nelle società europee di oggi e, all'indietro, le donne comuni, quelle aristocratiche e le principesse dell'età augustea.

I tre obiettivi formativi non scritti e non detti, tutti in controtendenza rispetto all'oggi, sono pensati in aula per i futuri docenti, un domani in classe per gli alunni: calarsi nella lentezza del tempo storico antico (la contro-immagine è la velocità del presente); educarsi alla complessità (la contro-immagine è la banalizzazione e l'estrema semplificazione), abituarsi alla formulazione di quesiti storici come cittadini (la controimmagine è l'indebolimento dell'etica civica al quale tutti assistiamo). 


\section{L'esperienza di Unimol per la storia moderna: storia e digital humanities, una proposta didattica}

Le motivazioni che hanno indotto il dipartimento di Scienze umane sociali e della formazione dell'Università degli Studi del Molise ad attivare, o meglio, a riattivare un corso di didattica della storia a partire dall'anno accademico 2016-2017 sono da far risalire a una pluralità di fattori, collegati tra loro dall'oggettivo riscontro di una lacuna nell'offerta formativa e didattica. Alla mancanza di un corso che all'interno del piano di studi della Laurea in scienze della formazione affrontasse in maniera specifica il tema dell'insegnamento della storia e delle sue metodologie, si è accompagnato il tentativo di rispondere a una oggettiva difficoltà per i futuri insegnanti riguardo all'insegnamento di una materia complessa, sistematica, interdisciplinare come la storia, questione tanto più delicata se si pensa che all'interno dei corsi istituzionali di storia medievale, moderna e contemporanea non sono previsti crediti relativi alla didattica. Alcune criticità relative all'organizzazione di un piano formativo coerente ed efficace nel formare i futuri insegnanti sono dovute, come sottolineato da Walter Panciera ed Enrico Valseriati, all'attuale assetto complessivo universitario italiano (Panciera e Valseriati 2019). In questo senso, la didattica della storia si è spesso trovata ai margini del sistema formativo, sia da un punto di vista strettamente culturale che da quello del suo inquadramento all'interno del panorama didattico, ciò anche come effetto indesiderato del suo eclettismo: basti pensare che la disciplina non afferisce direttamente, non del tutto a torto, ad alcun settore scientifico disciplinare. Più in generale, alcune vulnerabilità e arretratezze del sistema dell'istruzione italiana hanno contribuito a penalizzare la didattica della storia, come dimostra la carenza endemica di centri di ricerca, strutture, dottorati di ricerca, collane, riviste e volumi dedicati (Cajani 2019). A queste problematicità fa da contraltare però un vivace dibattito tra insegnanti e studiosi, che ha avuto fertili ripercussioni nelle discussioni su natura, contenuti, metodologia e missione della didattica della storia, collegate a una serie di 
veloci e radicali trasformazioni contingenti e al tentativo di rispondervi il più rapidamente possibile. Tra queste innovazioni radicali appare centrale la questione dei luoghi e modi di scoperta e fruibilità dell'informazione storica da parte delle generazioni più giovani: tecnologia digitale e informatizzazione spingono infatti sempre più spesso la storia fuori dalla scuola e dal suo strumento tradizionale di conoscenza, il manuale e, più in generale, il testo stampato.

Davanti a questi profondi mutamenti indotti dalla contingenza storica, la tipologia di corso che è stata messa in campo ha dovuto anche fare i conti con una ulteriore peculiarità. Pensato come insegnamento libero all'interno delle attività didattiche del dipartimento, esso ha attratto anche studenti di lettere, beni culturali e scienze della comunicazione, e ha visto la frequenza, attiva e partecipata, dunque, di futuri insegnanti con diversi ambiti e classi d'insegnamento. Per questo motivo, il corso ha assunto necessariamente un taglio più ampio rispetto alla questione della didattica della storia riservata esclusivamente alla scuola primaria, cercando di mettere in campo non soltanto le tradizionali lezioni frontali, ma introducendo gli studenti alla sensibilità e alla pratica del laboratorio storico, che non costituisce semplicemente uno spazio attrezzato, ma rappresenta un ambito di connessione tra la partecipazione attiva dello studente al processo della produzione della conoscenza storica e il rapporto tra tale pratica e una serie di competenze (Monducci 2018). Alla base del corso si è deciso di porre la centrale questione del legame tra il perché insegnare storia e il come insegnarla. Si tratta di due specifici temi che, pur conservando un'autonomia di fondo, non possono essere pensati singolarmente, pena una distorta rappresentazione non solo dell'ambito della disciplina ma anche della sua metodologia di insegnamento. Perché e come insegnare storia si trovano infatti in un rapporto di strutturata complementarità, che unisce in maniera inscindibile le questioni della didattica con quelle etiche e valoriali (Guarracino 2018). Fermo restando che le finalità etiche e valoriali potrebbero essere raggiunte anche prescindendo dall'insegnamento della storia e che fornire 
conoscenze senza la sensibilità a riconoscere la dimensione della storicità può comunque formare insegnanti, per quanto dimidiati nella loro coscienza e sensibilità storica, tuttavia tra le finalità etiche e politiche che si sono da sempre poste alle basi dell'insegnamento storico vi sono l'obiettivo della costruzione di una identità nazionale condivisa e, come sottolineato da Guarracino, più recentemente, la subordinazione della storia a scopi legati alla contingenza politica. Acquisita l'importanza della dimensione della storicità, dunque di un'esistenza oggettiva indipendente dal singolo, ci si trova davanti a un apparente paradosso: dover spiegare ai futuri docenti che pensare di insegnare tutta la storia è illusorio, e che bisognerà selettivamente domandarsi non solo come, ma anche - e preventivamente nell'organizzazione della didattica - quale storia insegnare. Si tratta di un articolatissimo dibattito che prende le mosse dal tradizionale eurocentrismo della storiografia, e che apre alla possibilità non solo di integrare il tradizionale approccio politicoistituzionale con diversi filoni storiografici quali la storia di genere, la storia economica, sociale, locale, nazionale, ma che rimanda anche alla decisione su quali siano i contenuti più adatti a essere trasmessi, ovvero quegli elementi di storia che, in combinazione con la metodologia didattica, contribuiscano in maniera decisiva a costruire la sensibilità e la consapevolezza cruciale insite nel senso della storicità.

Per realizzare questi obiettivi, le 48 ore del corso sono state divise in due parti, una prima basata su una discussione di base per introdurre concetti, metodi, lessico ed elementi di base della disciplina storica, e una seconda in cui ci si è soffermati soprattutto sulla funzione del laboratorio come spazio critico e alla sua integrazione con le digital humanities. Tra i concetti di fondo, particolare attenzione è stata rivolta ai generi storiografici e alla centralità delle fonti storiche, alla loro individuazione, al riconoscimento, all'interpretazione critica dei documenti nel processo di produzione della conoscenza storica (Panciera 2016). Allo stesso modo sono stati affrontati il tempo storico e la sua periodizzazione, introducendo al rapporto tra tempi e spazi, e alla necessità 
di misurare e delimitare tempi, confrontandosi con le periodizzazioni della storia e il concetto di "lungo periodo", ovvero l'alternarsi delle civiltà su un arco cronologico di secoli e millenni (Braudel 1997). Questa parte del corso ha cercato di privilegiare anche il lavoro di discussione e analisi in gruppi, nonché la formazione e il riconoscimento di un lessico politico, sociale e istituzionale di base in grado di definire e trasmettere il senso di storicità oggettiva e di appartenenza a una e più comunità sociali, politiche e culturali ben definite nei loro ambiti autonomi ma mai completamente separate l'una dall'altra. In conclusione, si è fatto cenno al dibattito sull'uso pubblico della storia e alla public history (Gallerano 1995), richiamando il tema delle finalità etiche dell'insegnamento della storia, e infine all'utilizzo della storia al di fuori dalle sedi scientifiche, nel dibattito pubblico, nella cultura e nei mezzi di comunicazione di massa.

La seconda parte del corso si è invece concentrata sulla pratica laboratoriale, la sua peculiare forma mentis (Mattozzi 2018) e le sue possibili e feconde interconnessioni con le digital humanities, le sue implementazioni e i suoi software di sviluppo (Foka 2017). Il laboratorio non è, come visto, un mero spazio attrezzato, quanto un metodo dinamico di apprendimento in cui lo studente può, grazie al suo ruolo attivo, superare la visione rigida di una conoscenza storica fatta da aneddoti slegati, sciolti nel loro ordine e senza alcun apparente senso logico. Attribuire un ruolo centrale alle digital humanities nella didattica della storia, a mio parere, può essere una mossa decisiva nel riannodare il filo con la generazione di studenti che, nati a cavallo del nuovo millennio, sono destinati a diventare i prossimi studenti universitari. Nati e cresciuti in un ambiente digitalizzato, possiedono naturalmente una forma mentis e una propensione diversa a pensare al rapporto tra presente e passato, priva dagli schemi interpretativi della realtà e del presente che hanno permeato il XIX e il XX secolo (Guarracino 2018): è diverso dunque, per loro, l'intero rapporto con realtà e testi. La vorticosità dei cambiamenti in ogni aspetto della realtà spinge queste generazioni a non cercare 
continuità con il passato e a non vedere nella storia uno strumento interpretativo per il presente e per assumere atteggiamenti dinanzi a esso, tanto che Serge Gruzinski è giunto a porre una provocatoria domanda alla comunità scientifica, domandandosi se ci fosse ancora bisogno della storia dinanzi a questa radicale mutazione (Gruzinski 2015). In questo senso le digital humanities rappresentano un deciso passo in avanti rispetto a Ict (Information communication technology), WebQuest (Ipertesti online, Power Point), Lim o al più basilare utilizzo di tablet e pc nella didattica. Le digital humanities e i suoi innumerevoli campi di applicazione e coinvolgimento attivo nell'apprendimento possono permettere di sviluppare più agevolmente la capacità di leggere le tracce di un mondo che sembra non essere più esistente ed entrare in contatto con fonti e documenti altrimenti irraggiungibili. Si è lavorato sull'ipotesi di un vero e proprio laboratorio di informatica umanistica, per permettere a futuri insegnanti di prendere contatto con le tecnologie e i metodi delle digital humanities e con software quali Omeka ${ }^{5}$, per fornire una base per leggere e operare su risorse, fonti, immagini, dati all'interno di un'interconnessione tra informatica-scienze umane-didattica, nella prospettiva delle storie interconnesse e dei network.

Questi approcci metodologici, concepiti all'interno di un sistema unitario e al tempo stesso dinamico e partecipato, aperto all'integrazione tra strumenti e metodi tradizionali quali i sussidiari, i manuali, la tradizionale e talora ritenuta vetusta lezione frontale, assieme allo sviluppo, in termini di sensibilità e competenze, della pratica laboratoriale, possono contribuire in maniera fattiva a rinnovare e ridefinire la formazione iniziale e in itinere degli insegnanti di storia. In questo ambito, un ruolo decisivo potrà essere giocato non solo dall'utilizzo delle nuove tecnologie, ma anche dalla costruzione di una rete di conoscenze, proposte e metodologie che, basandosi sulle digital humanities e sulle loro potenzialità di sviluppo, circolazione e connessione tra idee, progetti e prospet- 
tive di studio e ricerca, possa costruire un modello integrato di senso storico e di rappresentazione della dimensione della storicità oggettiva. L'idea di fondo di questo progetto è che per la formazione iniziale e in itinere degli insegnanti sia necessario conoscere lessico e concetti di base della disciplina storica, nonché avere chiaro il processo di produzione della conoscenza e il rapporto tra fonti e documenti, sfruttando le capacità di trasmissibilità e interazione attraverso strumenti e software digitali che privilegiano l'interconnessione tra luoghi, idee, prospettive di ricerca e di analisi critica delle fonti e dei fatti. In questa prospettiva tutti gli strumenti, tradizionali e innovativi, concepiti e usati in maniera attiva, concorrono alla formazione dei futuri docenti. Anzi, solo il loro utilizzo integrato e l'apertura all'interconnessione digitale, dunque a uno spazio dinamico e permanente di confronto, interazione e sviluppo critico, libero da letture ideologiche degli strumenti e delle forme della didattica, può sostenere in modo proficuo il percorso di arricchimento d'esperienza e modalità d'insegnamento della storia.

\section{Bibliografia di riferimento}

Bevilacqua, Piero. 1997. Sull'utilità della storia per l'avvenire delle nostre scuole. Roma: Donzelli.

Braudel, Fernand. 1997. "Histoire, mesure du monde." In Les ambitions de l'Histoire, di Fernand Braudel, 13-83. Paris: Éd. de Fallois (trad. it. 1998. Storia, misura del mondo. Bologna: il Mulino).

Cajani, Luigi. 2019. "Le vicende della Didattica della Storia in Italia." In Prospettive per la Didattica della Storia in Italia e in Europa, a cura di Enrico Valseriati, 121-30. Palermo: New Digital Frontiers.

Ceserani, Giovanna, Caroline Winterer, Dan Edelstein, Paula Findlen, e Nicole Coleman. 2017. "Historical Research in a Digital Age: Reflections from the Mapping the Republic of Letters Project." The American Historical Review 122, 2 (April): 40024. https://doi.org/10.1093/ahr/122.2.400.

Donato, Maria Pia. 2014. "Il patrimonio e le sfide di una storia interconnessa. A proposito del libro di T. Montanari, Istruzioni per l'uso del futuro. Il patrimonio culturale e la democrazia che verrà (Roma, Minimum Fax, 2014)." Giornale di storia 14. 
http://www.giornaledistoria.net/wp-content/uploads/2017/04/Content20140922_ MariaPiaDonatoInterventowordultimoDEF.pdf, consultato il 19.03.2020.

Foka, Anna, Jonathan Westin, e Adam Chapman. 2018. "Introduction to the DHQ Special Issue: Digital Technology in the Study of the Past." DHQ. Digital Humanities Quarterly 12 (2).

Gallerano, Nicola. 1995. L'uso pubblico della storia. Milano: FrancoAngeli.

Gruzinski, Serge. 2015. L'histoire pour quoi faire?. Paris: Fayard (trad. it. 2016. Abbiamo ancora bisogno della storia? Il senso del passato in un mondo globalizzato. Milano: Cortina).

Guarracino, Scipione. 20183. “Le questioni dell'insegnare storia.” In Insegnare storia. Il laboratorio storico e altre pratiche attive, a cura di Francesco Monducci, 3-12. Torino: Utet.

Hasberg, Wolfgang. 2019. "Academic profiling and orientation on practice. Balancing Act of History Didactics and History Teacher Education in Germany." In Prospettive per la Didattica della storia in Italia e in Europa, a cura di Enrico Valseriati, 155-80. Palermo: New Digital Frontiers.

Mattozzi, Ivo. 2003. "La didattica laboratoriale nella modularità e nel curricolo di storia." Quaderni di Clio '92 4 (dicembre): 41-54. http://storiairreer.it/materiali/materiali/metodistrategietecniche/mattozzilaboratorio3protetto.pdf.

_ _ 20183. "La mente laboratoriale." In Insegnare storia. Il laboratorio storico e altre pratiche attive, a cura di Francesco Monducci, 143-52. Torino: Utet.

Monducci, Francesco. 20183. "Il manuale, per una didattica attiva." In Insegnare storia. Il laboratorio storico e altre pratiche attive, a cura di Francesco Monducci, XIX-XXIV. Torino: Utet.

Panciera, Walter. 2016. Insegnare storia nella scuola primaria e dell'infanzia. Roma: Carocci.

Panciera, Walter e Enrico Valseriati. 2019. "La didattica della storia in Italia e in Europa." In Prospettive per la Didattica della Storia in Italia e in Europa, a cura di Enrico Valseriati, 7-14. Palermo: New Digital Frontiers.

Panciera, Walter e Andrea Zannini. 20133. Didattica della storia. Manuale per la formazione degli insegnanti. Firenze: Le Monnier.

Ricuperati Giuseppe. 2005. Apologia di un mestiere difficile. Problemi, insegnamenti e responsabilità della storia. Roma-Bari: Laterza. 
